\title{
$\omega$-limit sets for maps of the interval
}

\author{
LOUIS BLOCK AND ETHAN M. COVEN \\ Department of Mathematics, University of Florida, Gainesville, FL 32611, USA; \\ Department of Mathematics, Wesleyan University, Middletown, CT 06457, USA
}

(Received 20 June 1985)

Abstract. Let $f$ denote a continuous map of a compact interval to itself, $P(f)$ the set of periodic points of $f$, and $\Lambda(f)$ the set of $\omega$-limit points of $f$. Sarkovskri has shown that $\Lambda(f)$ is closed, and hence $\overline{P(f)} \subseteq \Lambda(f)$, and Nitecki has shown that if $f$ is piecewise monotone, then $\Lambda(f)=\overline{P(f)}$. We prove that if $x \in \Lambda(f)-\overline{P(f)}$, then the set of $\omega$-limit points of $x$ is an infinite minimal set. This result provides the inspiration for the construction of a map $f$ for which $\Lambda(f) \neq \overline{P(f)}$.

\section{Introduction}

Let $f$ denote a continuous map of a compact interval $I$ to itself, $P(f)$ the set of periodic points of $f$, and $\Lambda(f)=\bigcup_{x \in I} \omega(x)$, where $\omega(x)$ denotes the set of $\omega$-limit points of $x$. A. N. Sarkovskiii [S1] has shown that $\Lambda(f)$ is closed, and hence that $P(f) \subseteq \Lambda(f)$. Additional results concerning $\omega$-limit sets for maps of the interval have been obtained by Sarkovskǐi and H. K. Kenzegulov [SK].

In this paper, we consider the following problem: Is $\Lambda(f)=\overline{P(f)}$ ?

Z. Nitecki [N1] has shown that if $f$ is piecewise monotone, then $\Lambda(f) \subseteq \overline{P(f)}$, so that in this case $\Lambda(f)=\overline{P(f)}$.

We attack the problem by assuming that $\Lambda(f) \neq \overline{P(f)}$ and seeing what follows. We obtain the following result.

THEOREM. If $x \in \Lambda(f)-\overline{P(f)}$, then $\omega(x)$ is an infinite minimal set.

This theorem and more specific results obtained in the course of its proof provide the inspiration for the construction of an example where $\Lambda(f) \neq P(f)$. In this example, there is a point $x \in \Lambda(f)-\overline{P(f)}$ such that $f \mid \omega(x)$ is topologically conjugate to the group translation known as the 'adding machine.'

This example, incidentally, has the following properties:

(1) $P(f)$ consists of exactly one periodic orbit of period $2^{n}(n=0,1,2, \ldots)$.

(2) $f \mid \Omega(f)$ is not one-to-one, where $\Omega(f)$ denotes the set of non-wandering points of $f$.

Thus the assertion of Sarkovskiri [S2, theorem B], that if all periodic points have period a power of two, then $f \mid \Omega(f)$ is a homeomorphism, is false.

In the example above, the restriction of $f$ to $\omega(x)$ is topologically conjugate to a group translation. We give a second example to show that this need not always be the case. In fact, this example shows that $f \mid \omega(x)$ need not be one-to-one when $x \in \Lambda(f)-\overline{P(f)}$. 
Finally, we give a third example to show that the theorem does not hold when $\Lambda(f)$ is replaced by $\Omega(f)$, i.e. if $x \in \Omega(f)-\widehat{P(f)}$, then $\omega(x)$ need not be a minimal set. This example also yields an easy construction of a map $f$ having a point $x \in \Omega(f)-\overline{P(f)}$ with an infinite orbit. An example of this phenomenon was first constructed by the second author and Nitecki [CN].

This work was done while the first author was J. H. Van Vleck Visiting Professor of Mathematics at Wesleyan University.

\section{The theorem and its proof}

We begin with some notation. Recall that $f$ denotes a continuous map of a compact interval $I$ to itself.

Let $x \in I$. Let orb $(x)$ denote the orbit of $x$. By an $R$-neighbourhood of $x$ we will mean an interval $[x, x+\varepsilon]$ where $\varepsilon>0$, and by an $L$-neighbourhood of $x$ we will mean an interval $[x-\varepsilon, x]$ where $\varepsilon>0$. If $p$ is a fixed point of $f$ and $S$ is $R$ or $L$, we let $W^{u}(p, f, S)$ denote the set of $y \in I$ such that for any $S$-neighbourhood $V$ of $p, y \in f^{k}(V)$ for some positive integer $k$.

LEMMA 1. If $x \in \Lambda(f)$ and $x$ has a finite orbit, then $x \in \overline{P(f)}$.

Proof. Since $\Lambda(f)=\Lambda\left(f^{n}\right)$ and $P(f)=P\left(f^{n}\right)$, we may assume that $f(x)$ is a fixed point $p$ of $f$. We may also assume that $x \neq p$, otherwise there is nothing to prove. Finally, without loss of generality, we may assume that $x<p$. Let $y \in I$ with $x \in \omega(y)$.

Case 1 . There is a strictly increasing sequence $\left(n_{i}\right)$ of positive integers such that the sequence $\left(f^{n_{i}}(y)\right)$ is strictly decreasing and approaches $x$.

Let $i$ be a positive integer. Then $f^{n_{i+1}}(y)<f^{n_{i}}(y)$, and hence $f^{n_{i+1}-n_{i}}\left(f^{n_{i}}(y)\right)<f^{n_{i}}(y)$. Since $f^{n_{i+1}-n_{i}}(x)=p>x$, this implies that there is a periodic point in $\left(x, f^{n_{i}}(y)\right)$. It follows that $x \in \overline{P(f)}$.

Case 2. There is a strictly increasing sequence $\left(n_{i}\right)$ of positive integers such that the sequence $\left(f^{n_{i}}(y)\right)$ is strictly increasing and approaches $x$.

There is a side $S$ of $p$ such that a subsequence of $\left(f^{n_{i}+1}(y)\right)$ approaches $p$ from this side. It follows that $x \in W^{u}(p, f, S)$ and thus, since $W^{u}(p, f, S)$ is connected, that $[x, p] \subseteq W^{u}(p, f, S)$.

We claim that some element of the orbit of $y$ is in $W^{u}(p, f, S)$. Since the claim follows immediately if $S=L$, we will assume in proving the claim that $S=R$. Also, if either $x$ or $p$ is an interior point of $W^{u}(p, f, R)$, then the claim follows. Thus to prove the claim, we may assume that $W^{u}(p, f, R)=[x, p]$.

Since $f$ is uniformly continuous, there is a $\delta>0$ such that if $z_{1}$ and $z_{2}$ are points in $I$ with $\left|z_{1}-z_{2}\right|<\delta$, then $\left|f\left(z_{1}\right)-f\left(z_{2}\right)\right|<p-x$. Let $v \in I$ with $p<v<p+\delta$. Since $v \notin W^{u}(p, f, R)$, there is a $\gamma>0$ such that $v \notin \bigcup_{n=0}^{\infty} f^{n}([p, p+\gamma])$. Now, for some positive integer $j, f^{j}(y) \in(p, p+\gamma)$. On the other hand, for some integer $m>j$, $f^{m}(y)<x$. This implies that for some integer $k$ with $j<k<m, f^{k}(y) \in(x, p)$. This establishes the claim.

Since $W^{u}(p, f, S)$ is invariant under $f$, it follows from the claim that $x$ is in the interior of $W^{u}(p, f, S)$. Let $K$ be an $L$-neighbourhood of $x$ with $K \subseteq W^{u}(p, f, S)$. 
Then $f(K)$ contains an $S$-neighbourhood of $p$, and hence $f^{j}(K) \supseteq K$ for some positive integer $j$. Thus $x \in \overline{P(f)}$.

We now adopt some notation and make a standing hypothesis for the next three lemmas. Let $J$ denote a component of $I-\overline{P(f)}, c$ the left endpoint of $J$, and $d$ the right endpoint of $J$. We also assume that there is a point $z \in J$ and a positive integer $m$ such that $f^{m}(z) \in J$ and $f^{m}(z)>z$.

LEMma $2([\mathrm{C}],[\mathbf{X}])$. If $y \in J$ and $f^{n}(y) \in J$ for some positive integer $n$, then $f^{n}(y)>y$.

Proof. Let $g=f^{m}$, and observe that the interval $[z, g(z)]$ contains no periodic points of $g$. It follows easily by induction that $g^{k}(z)>z$ for every positive integer $k$. In particular, $f^{m n}(z)>z$. If $f^{n}(y)<y$, then a similar argument would show that $f^{m n}(y)<y$. Then $f^{m n}$ would have a fixed point between $y$ and $z$, a contradiction. Thus $f^{n}(y)>y$.

LEMMA $3([\mathrm{C}])$. If $x \in(c, d)$ is non-wandering and has an infinite orbit, then the sets $H, f(H), f^{2}(H), \ldots$ are pairwise disjoint, where $H=[x, d]$.

Proof. If the sets $f^{k}(H)$ are not pairwise disjoint, then $E=\bigcup_{k=0}^{\infty} f^{k}(H)$ has finitely many components. Since $x$ has an infinite orbit, this implies that $f^{N}(x)$ is in the interior of $E$ for some positive integer $N$. Since $x$ is non-wandering, there are sequences of points $x_{k} \rightarrow x$ and positive integers $n_{k} \rightarrow \infty$ with $f^{n_{k}}\left(x_{k}\right)=x$ [CN, lemma 2.6]. Now, for $k$ sufficiently large, $n_{k}>N$ and $f^{N}\left(x_{k}\right) \in E$. Thus $x=f^{n_{k}-N}\left(f^{N}\left(x_{k}\right)\right)=$ $f^{j}(y)$ for some $y \in H$ and some positive integer $j$. Hence $f^{j}(v)<v$ and $f^{j}(v) \in J$ for some $v \in(x, d)$. This contradicts lemma 2 .

LEMMA 4. If $x \in(c, d) \cap \Lambda(f)$, then $d \in \omega(x)$.

Proof. Let $z_{f}$ denote the greatest lower bound of $\{y \in$ orb $(x): y>x\}$. It follows from the standing hypothesis that this set is non-empty. By lemma $3, z_{f} \geq d$ and $d$ is not an element of orb $(x)$. Thus to prove the lemma, it suffices to show that $z_{f}=d$. We will assume that $z_{f}>d$ and obtain a contradiction.

There is a periodic point $p \in\left[d, z_{f}\right]$. Let $n$ be the period of $p$, and let $g=f^{n}$. Note that since $\Lambda(f)=\Lambda(g)$ and $P(f)=P(g), x \in \Lambda(g)$ and $J$ is a component of $I-\overline{P(g)}$. It follows from lemma 2 and the fact that $x \in \Lambda(g)$ that the standing hypothesis holds for $g$.

For $y \in I$, let $\operatorname{orb}^{\prime}(y)$ denote the orbit of $y$ under $g$ and $\omega^{\prime}(y)$ the set of $\omega$-limit points of $y$ under $g$. Let $z_{g}$ denote the greatest lower bound of $\left\{y \in \operatorname{orb}^{\prime}(x): y>x\right\}$. Then $z_{g} \geq z_{f}$.

Let $W^{u}(x, g)$ denote the set of $y \in I$ such that for every neighbourhood $V$ of $x$, $y \in g^{k}(V)$ for some positive integer $k$. Then $f\left(W^{u}(x, g)\right) \subseteq W^{u}(x, g)$ and by lemma $2,(c, x) \cap W^{u}(x, g)=\varnothing$. Now $x \in \omega^{\prime}(y)$ for some $y \in I$. Again by lemma 2 , $\operatorname{orb}^{\prime}(y) \cap(x, d)=\varnothing$. Thus $\left[x, z_{g}\right] \subseteq W^{u}(x, g)$.

Let $A$ be the component of $W^{u}(x, g)$ which contains $x$. Then $A$ is an interval with left endpoint $x, p \in A$, and $g(A) \subseteq A$. Hence there is a positive integer $j \leq 2$ such that $g^{j}(x)$ is in the interior of $A$. Let $K$ be a neighbourhood of $g^{j}(x)$ with $K \subseteq A$, and let $V$ be a neighbourhood of $x$ with $V \subseteq J, g(V) \cap V=\varnothing$, and $g^{j}(V) \subseteq K$. 
For any $v \in V$ and any positive integer $k, g^{k}(v) \geq x$. It follows from this and lemma 3 that $x \notin \omega^{\prime}(y)$, which is a contradiction.

THEOREM. If $x \in \Lambda(f)-\overline{P(f)}$, then $\omega(x)$ is an infinite minimal set.

Proof. Let $J$ be the component of $I-\overline{P(f)}$ which contains $x$, and let $c$ and $d$ denote the left and right endpoints of $J$. By [CN, lemma 2.7], $x$ is not an endpoint of the ambient interval $I$; hence $x \in(c, d)$.

Since $x \in \Lambda(f)$, there is a point $z \in J$ and a positive integer $m$ such that $f^{m}(z) \in J$. Without loss of generality we may assume that $f^{m}(z)>z$. By lemma $1, x$ has an infinite orbit, and by lemma $4, d \in \omega(x)$. It follows from lemma 3 that $d$ has an infinite orbit and that $\omega(x)=\omega(d)$. Since $\omega(x)$ is a closed invariant set, it follows that $\omega(x)=\overline{\text { orb }(d)}$. Thus to prove the theorem, it suffices to show that orb $(d)$ is a minimal set.

A classical result of G. D. Birkhoff $[B$, p. 199] states that $\overline{\text { orb }(d)}$ is a minimal set if and only if $d$ is almost periodic, i.e. for every neighbourhood $V$ of $d$, the orbit of $d$ returns to $V$ with bounded gaps. We complete the proof by proving the stronger statement:

(*) For every $\varepsilon>0$, there is a positive integer $n$ such that $f^{j n}(d) \in(d, d+\varepsilon)$ for every positive integer $j$.

Let $\varepsilon>0$. For some $y \in(c, d), x \in \omega(y)$. It follows from lemmas 2 and 3 that orb $(y) \cap[x, d]=\varnothing$. Since $d \in \omega(x), f^{M}(y) \in(d, d+\varepsilon)$ for some positive integer $M$. Also, since $x \in \omega(y), f^{K}(y) \in(c, x)$ for some integer $K>M$.

Since $f^{K+i}(y) \in(c, x)$ for some positive integer $i$, it follows from lemmas 2 and 3 that $f^{i}\left(\left[f^{K}(y), x\right]\right)$ contains an interval $[d, d+\gamma]$, where $\gamma>0$ and $d+\gamma<f^{M}(y)$. There is a periodic point $p \in(d, d+\gamma)$. Let $n$ be the period of $p$.

Let $j$ be any positive integer. We must show that $f^{\text {jn }}(d) \in(d, d+\varepsilon)$.

First, suppose that $f^{j n}(d) \geq d+\varepsilon$. Then some power of $f$ maps $[d, p]$ to an interval containing $f^{M}(y)$. Hence some (other) power of $f$ maps $\left[f^{K}(y), x\right]$ to an interval containing $f^{K}(y)$. This contradicts lemma 2.

Finally, suppose that $f^{j n}(d) \leq d$. By lemmas 2 and 3 and the continuity of the powers of $f, f^{j n}(d) \leq c$. Hence $f^{j n}([d, p]) \supseteq[c, p]$, and again some power of $f$ maps $\left[f^{K}(y), x\right]$ to an interval containing $f^{K}(y)$, a contradiction.

This proves $(*)$ and hence the theorem.

\section{Examples}

Example 1. We construct a continuous map $f$ of the interval with a point $x \in$ $\Lambda(f)-\overline{P(f)}$. According to the theorem, $\omega(x)$ must be an infinite minimal set. In this example, the restriction of $f$ to $\omega(x)$ is topologically conjugate to the 'adding machine', i.e. translation by +1 on the group of 2 -adic integers. We briefly review the construction of the adding machine and its realization on the interval.

The 2-adic integers $A$ is the topological group (based on the Cantor set) consisting of all formal expressions $\sum_{i=0}^{\infty} a_{i} 2^{i}$ where $a_{i}$ is 0 or 1 . We may think of the non-negative integers as a subset of $\boldsymbol{A}$ by associating with each non-negative integer its base 2 expansion. Addition in $A$ is the extension of the usual addition on the non-negative 
integers to $A$, i.e. with carrying. The adding machine is the map $p: A \rightarrow A$ defined by $p(a)=a+1$. It is well-known that $A$ is a minimal set.

We may identify $A$ with the Cantor middle third set $C$ by identifying $\sum_{i=0}^{\infty} a_{i} 2^{i}$ with the real number $\sum_{i=0}^{\infty} 2 a_{i} 3^{-i-1}$. Notice that

$$
C \subseteq\left[0, \frac{1}{3}\right] \cup\left[\frac{2}{3}, \frac{7}{9}\right] \cup\left[\frac{8}{9}, \frac{25}{27}\right] \cup \cdots \cup\{1\} .
$$

We define a map $f$ on the set on the right as follows. Let $f$ map $\left[0, \frac{1}{3}\right],\left[\frac{2}{3}, \frac{7}{9}\right],\left[\frac{8}{9}, \frac{25}{27}\right], \ldots$ by translation onto $\left[\frac{2}{3}, 1\right],\left[\frac{2}{9}, \frac{1}{3}\right],\left[\frac{2}{27}, \frac{1}{9}\right], \ldots$ and let $f(1)=0$. It can be easily verified that $f(C)=C$ and that $f \mid C$ is topologically conjugate to the adding machine.

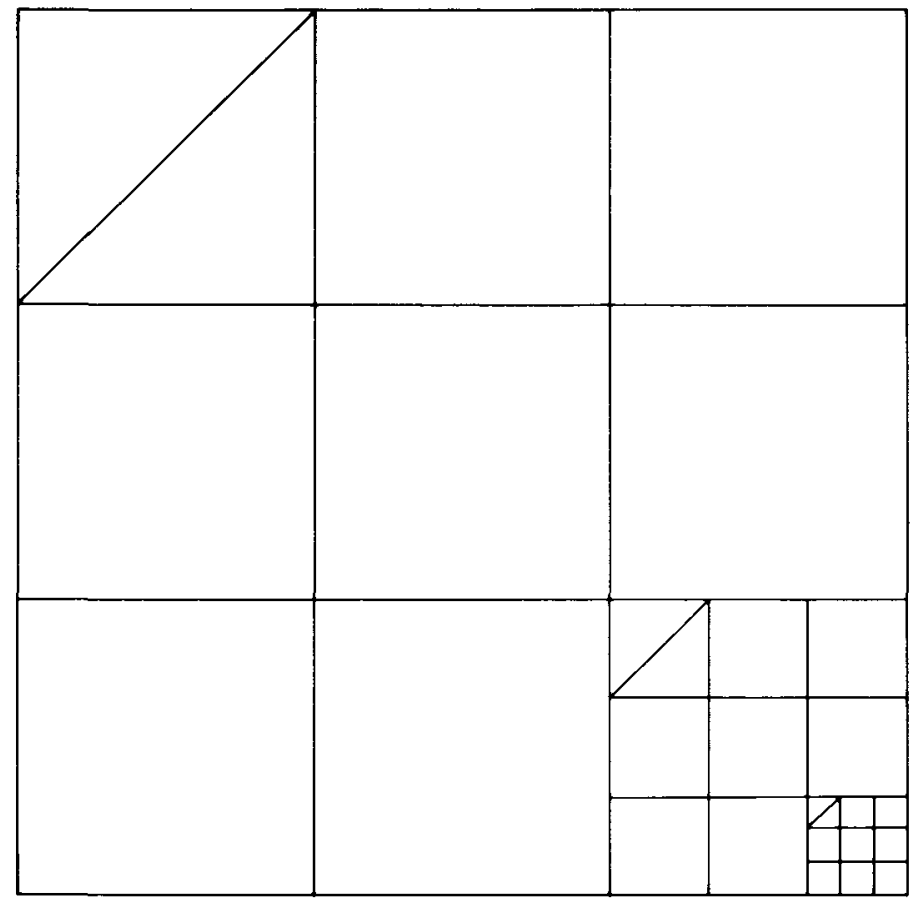

FIgure 1

We remark that to this point our construction is the same as that used by J. P. Delahaye [D] to construct a map of the interval having exactly one periodic orbit of period $2^{n}(n=0,1,2, \ldots)$ and no other periodic orbits. Notice that no matter how we extend $f$ to a map of an interval $I \supseteq[0,1], f \mid C$ will be topologically conjugate to the adding machine and $\omega(0)$ will be $C$.

We will extend $f$ to the interval $[-1,2]$ in such a way that:

(1) $-\frac{2}{3} \in \Lambda(f)-\overline{P(f)}$.

(2) $f\left(-\frac{2}{3}\right)=f(0)$, and hence $\omega\left(-\frac{2}{3}\right)=C$.

To extend $f$ to the interval $[-1,2]$, it suffices to define $f$ on the intervals $[-1,0]$, $[1,2]$ and $I_{1}=\left[\frac{1}{3}, \frac{2}{3}\right], I_{2}=\left[\frac{7}{9}, \frac{8}{9}\right], I_{3}=\left[\frac{25}{27}, \frac{26}{27}\right], \ldots$.

First, we let $f(x)=1-x$ on the interval $[1,2]$. Then $f$ maps $[1,2]$ linearly onto $[-1,0]$, reversing order. Let $t_{0}=2, x_{0}=\frac{5}{3}$ (the unique point in $[1,2]$ satisfying $\left.f\left(x_{0}\right)=-\frac{2}{3}\right)$ and $s_{0}=\left(\frac{2}{3}\right) x_{0}+\left(\frac{1}{3}\right) t_{0}=\frac{16}{9}$. 
Next, we define $f$ inductively on the intervals $I_{n}=\left[l_{n}, r_{n}\right] . f$ will have exactly one turning point in each $I_{n}$, the midpoint $t_{n}=1-1 / 2 \cdot 3^{n-1}$, and will be linear on $\left[l_{n}, t_{n}\right]$ and $\left[t_{n}, r_{n}\right]$. Since $f$ is already defined on $l_{n}$ and $r_{n}$, it suffices to define $f$ on $t_{n}$.

Let $f\left(t_{1}\right)=s_{0}$, and (having extended $f$ to $\left[l_{1}, r_{1}\right]$ ) let $x_{1}$ be the unique point in $\left[l_{1}, t_{1}\right]$ satisfying $f\left(x_{1}\right)=x_{0}$. Let $s_{1}=\left(\frac{2}{3}\right) x_{1}+\left(\frac{1}{3}\right) t_{1}$. We repeat the procedure on $I_{2}$. Let $f\left(t_{2}\right)=s_{1}$, let $x_{2} \in\left[l_{2}, t_{2}\right]$ satisfy $f\left(x_{2}\right)=x_{1}$, and let $s_{2}=\left(\frac{2}{3}\right) x_{2}+\left(\frac{1}{3}\right) t_{2}$.

For the inductive step, we use the auxiliary intervals $\left[l_{n}^{\prime}, t_{n}^{\prime}\right]=\left[1 / 3^{n}, 1 / 2 \cdot 3^{n-1}\right]$ $(n=2,3, \ldots)$. Note that $f^{2^{n-1}-1}$ is already defined on $\left[l_{n}^{\prime}, t_{n}^{\prime}\right]$ - in fact $\left[l_{n}^{\prime}, t_{n}^{\prime}\right]$, $f\left(\left[l_{n}^{\prime}, t_{n}^{\prime}\right]\right), \ldots, f^{2^{n-1}-2}\left(\left[l_{n}^{\prime}, t_{n}^{\prime}\right]\right)$ all lie in $\left[0, \frac{1}{3}\right] \cup\left[\frac{2}{3}, \frac{7}{9}\right] \cup \cdots-$ and that $f^{2^{n-1}-1}$ maps $\left[l_{n}^{\prime}, t_{n}^{\prime}\right]$ by translation onto $\left[l_{n}, t_{n}\right]$.

Assuming that $f\left(t_{n}\right), x_{n}$, and $s_{n}$ have already been defined, let $x_{n}^{\prime}$ and $s_{n}^{\prime}$ be the unique points in $\left[l_{n}^{\prime}, t_{n}^{\prime}\right]$ such that $f^{2^{n-1}-1}\left(x_{n}^{\prime}\right)=x_{n}$ and $f^{2^{n-1}-1}\left(s_{n}^{\prime}\right)=s_{n}$. Let $f\left(t_{n+1}\right)=$ $s_{n}^{\prime}$, let $x_{n+1}$ be the unique point in $\left[l_{n+1}, t_{n+1}\right]$ satisfying $f\left(x_{n+1}\right)=x_{n}^{\prime}$, and let $s_{n+1}=\left(\frac{2}{3}\right) x_{n+1}+\left(\frac{1}{3}\right) t_{n+1}$.

The construction is depicted in figure 2.

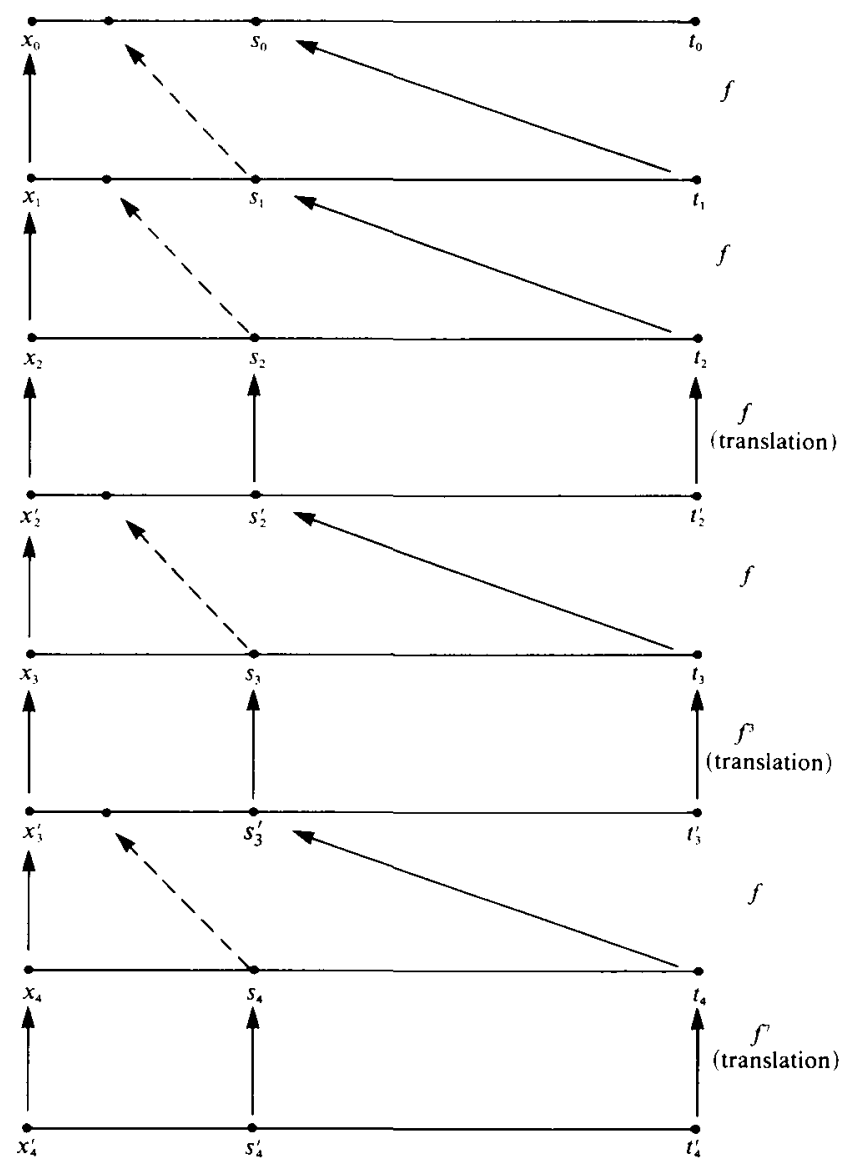

FIGURE 2 
The last step in the construction is to define $f$ on the interval $[-1,0]$. Let $f$ have the constant value $\frac{2}{3}$ on the interval $\left[-\frac{2}{3}, 0\right]$ and let $f(-1)$ be the unique point in $\left[\frac{1}{3}, \frac{2}{3}\right] \cap f^{-1}\left(\frac{1}{3}\right)$. (In case anyone cares, $f(-1)=\frac{55}{84}$.) Finally, let $f$ be linear on the interval $\left[-1,-\frac{2}{3}\right]$.

The graph of $f$ is displayed in figure 3 .

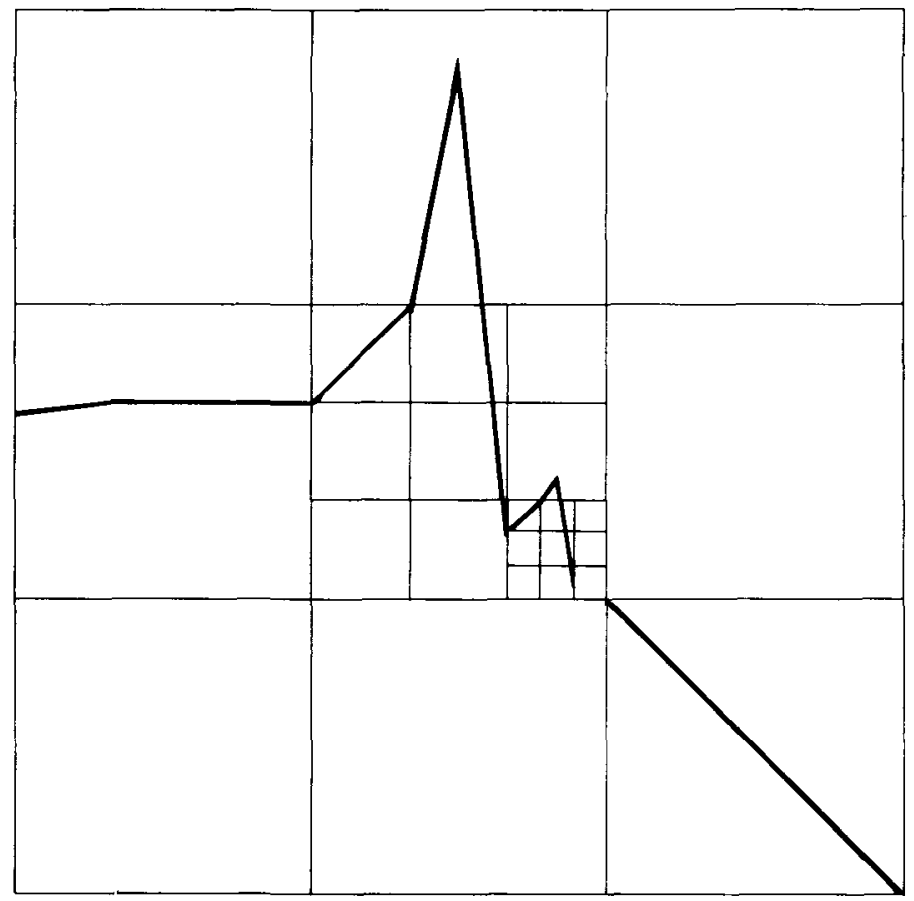

FIGURE 3

We will verify that $-\frac{2}{3} \in \Lambda(f)-\overline{P(f)}$. Consider the Cantor middle third set constructed on the interval $J=\left[-1,-\frac{2}{3}\right]$. By a deleted interval in $J$ we will mean a component of the complement of this set in $J$. We make the following observations.

(1) If $K$ is a deleted interval in $J$ of length $1 / 3^{n}$, then $K \subseteq\left(-1,-\frac{2}{3}-1 / 3^{n+1}\right)$.

(2) If $x \in J$ is not in a deleted interval, then $f^{k}(x) \geq 0$ for every positive integer $k$.

(3) If $x \in K$, for $K$ as in (1), then $f^{k}(x) \geq-\frac{2}{3}-1 / 3^{n+2}$ for every positive integer $k$. Note that (1) is obvious and that (2) follows immediately from the fact that $f^{2}$ maps $J$ linearly onto the interval $\left[\frac{2}{9}, \frac{1}{3}\right]$. (3) also follows from the construction of $f$, although not quite immediately. For example, suppose that $x \in K$, where $K$ is the deleted middle third of $J$, i.e. $K=\left(-\frac{8}{9},-\frac{7}{9}\right)$. Then $f^{2}(x) \in\left(\frac{7}{27}, \frac{8}{27}\right)$, the deleted middle third of $\left[\frac{2}{9}, \frac{1}{3}\right]$, and hence $f^{3}(x) \in\left(\frac{25}{27}, \frac{26}{27}\right)$. From this interval different orbits may take different paths to return to $J$. However, any such path must pass through $I_{1}$, and the only way a point can move from a deleted interval in $[0,1]$ of length $1 / 3^{n}$ to one of larger length is to pass through $I_{n}$ and then through $\left(l_{n-1}^{\prime}, s_{n-1}^{\prime}\right]$. Thus, in order for 
$x$ to return to $J$, we must have for some positive integer $k$

and finally

$$
\begin{aligned}
f^{k}(x) & \in\left(\frac{25}{27}, \frac{26}{27}\right) \\
f^{k+1}(x) & \in\left(\frac{1}{9}, s_{2}^{\prime}\right] \\
f^{k+2}(x) & \in\left(\frac{7}{9}, s_{2}\right] \\
f^{k+3}(x) & \in\left(\frac{1}{3},\left(\frac{2}{3}\right) x_{1}+\left(\frac{1}{3}\right) s_{1}\right] \\
f^{k+4}(x) & \in\left(1,\left(\frac{8}{9}\right) x_{0}+\left(\frac{1}{9}\right) s_{0}\right]=\left(1, \frac{5}{3}+\frac{1}{81}\right]
\end{aligned}
$$

$$
f^{k+5}(x) \in\left[-\frac{2}{3}-\frac{1}{81}, 0\right) \text {. }
$$

By following the orbit of $\left(-\frac{8}{9},-\frac{7}{9}\right)$ as above, we see that

$$
f^{8}\left(-\frac{8}{9},-\frac{2}{3}\right) \supseteq f^{8}\left(-\frac{8}{9},-\frac{7}{9}\right) \supseteq\left(-\frac{2}{3}-\frac{1}{81},-\frac{2}{3}\right) \supseteq\left(-\frac{2}{3}-\frac{2}{243},-\frac{2}{3}\right) \text {. }
$$

In the same way we obtain

(4) For every integer $i \geq 2$, there is a positive integer $n_{i}$ such that

$$
f^{n_{i}}\left(-\frac{2}{3}-2 / 3^{i},-\frac{2}{3}\right) \supseteq\left(-\frac{2}{3}-2 / 3^{i+3},-\frac{2}{3}\right) .
$$

Proposition. $-\frac{2}{3} \in \Lambda(f)-\overline{P(f)}$.

Proof. It follows from (1), (2), and (3) that $[-1,0] \cap P(f)=\varnothing$, and hence $-\frac{2}{3} \notin \overline{P(f)}$.

To see that $-\frac{2}{3} \in \Lambda(f)$, notice that by (4) there are closed intervals $K_{i}$ and positive integers $n_{i}(i=1,2,3, \ldots)$ such that $f^{n_{i}}\left(K_{i}\right) \supseteq K_{i+1}$ for each positive integer $i$ and $\bigcap_{i=1}^{\infty} K_{i}=\left\{-\frac{2}{3}\right\}$. Let $J_{1}=K_{1}, J_{2}=\left\{x \in J_{1}: f^{n_{1}}(x) \in K_{2}\right\}, J_{3}=\left\{x \in J_{2}: f^{n_{1}+n_{2}}(x) \in K_{3}\right\}$, etc. There is a point $y \in \bigcap_{n=1}^{\infty} J_{n}$, and $-\frac{2}{3} \in \omega(y)$.

The reader can verify that $f$ has exactly one periodic orbit of period $2^{n}$ ( $n=$ $0,1,2, \ldots)$ and no other periodic orbits. (Each interval $I_{n}$ contains a repelling periodic orbit of period $2^{n-1}$.) Since $f\left(-\frac{2}{3}\right)=f(0)$ and both points are non-wandering, this map provides a counterexample to the assertion of Sarkovskĭi [S2, theorem B] that if the only periods of periodic points are powers of two, then $f \mid \Omega(f)$ is a homeomorphism.

In example $1, \omega\left(-\frac{2}{3}\right)$ is a topological group and $f$ acts on it by translation. In particular, $f$ is one-to-one on $\omega\left(-\frac{2}{3}\right)$. This need not be the case. In the next example, we construct (following Nitecki $[\mathbf{N 2}, 4.18]$ ) a map $f^{\prime}$ with a point $x^{\prime} \in \Lambda\left(f^{\prime}\right)-\overline{P\left(f^{\prime}\right)}$ such that $f^{\prime} \mid \omega\left(x^{\prime}\right)$ is not one-to-one.

Example 2. We modify the map $f$ of example 1 by replacing every point in a backward orbit by an interval.

First, pick a point $z \in C$ (for example, $z=\frac{1}{4}$ ) such that every $R$-neighbourhood of $z$ and every $L$-neighbourhood of $z$ contains points of $C$ other than $z$. Next, let $\left[0^{\prime}, 1^{\prime}\right]$ be a copy of $[0,1]$, and let $C^{\prime} \subseteq\left[0^{\prime}, 1^{\prime}\right]$ be a perfect set whose components are in one-to-one order-preserving correspondence with those of $C$, such that for each integer $k \geq 0$, the component corresponding to the unique point in $f^{-k}(z) \cap C$ is a non-degenerate closed interval $J_{k}^{\prime}$, and all other components are points.

Let $f^{\prime}$ be the continuous map of $C^{\prime}$ to $C^{\prime}$ defined as follows.

(1) If $x \in C$ and $x \notin \bigcup_{k=0}^{\infty} f^{-k}(z)$, then $f^{\prime}\left(x^{\prime}\right)=(f(x))^{\prime}$.

(2) For each integer $k \geq 1, f^{\prime}$ maps $J_{k}^{\prime}$ linearly onto $J_{k-1}^{\prime}$, preserving order.

(3) $f^{\prime}$ collapses $J_{0}^{\prime}$ to the point $(f(z))^{\prime}$. 
Next, extend $f^{\prime}$ to the intervals $\left[0^{\prime},\left(\frac{1}{3}\right)^{\prime}\right],\left[\left(\frac{2}{3}\right)^{\prime},\left(\frac{7}{9}\right)^{\prime}\right], \ldots$ so that $f^{\prime}$ is linear on each deleted interval of $C^{\prime}$ which lies in any of these intervals. Notice that if $M^{\prime}$ denotes the set of boundary points of $C^{\prime}$, i.e. the points in $C^{\prime}$ not interior to any of the $J_{k}^{\prime}$, then $M^{\prime}$ is a minimal set and $f^{\prime}$ is not one-to-one on $M^{\prime}$. ( $f^{\prime}$ identifies the endpoints of $J_{0}^{\prime}$.)

Finally, extend $f^{\prime}$ to $\left[(-1)^{\prime}, 2^{\prime}\right]$ in the same way that $f$ was extended to $[-1,2]$. Then $\left(-\frac{2}{3}\right)^{\prime} \in \Lambda\left(f^{\prime}\right)-\overline{P\left(f^{\prime}\right)}$ and $\omega\left(\left(-\frac{2}{3}\right)^{\prime}\right)=M^{\prime}$.

Our third and final example shows that the theorem does not hold if we replace $\Lambda(f)-\widehat{P(f)}$ by $\Omega(f)-\widehat{P(f)}$. We construct a map $g:[-1,1] \rightarrow[-1,1]$ with a point $x \in \Omega(g)-\overline{P(g)}$ such that $\omega(x)$ is not a minimal set.

Example 3. Let $g(x)=3 x$ on the interval $\left[0, \frac{1}{3}\right]$ and $g(x)=3 x-2$ on the interval $\left[\frac{1}{2}, 1\right]$. If $C$ again denotes the Cantor middle third set, then $g(C)=C$ and $g \mid C$ is topologically conjugate in a natural way to the full one-sided shift of two symbols. In particular, for some $y \in C, \omega(y)=C$. (Any $y \in C$ whose ternary expansion contains every finite string of zeros and twos will do.)

Pick such a point $y$ and extend $g$ to $[-1,1]$ as follows. Let $g(-1)=0, g(x)=y$ for $x \in\left[-\frac{1}{2},-\frac{1}{4}\right]$, and let $g$ be linear on the intervals $\left[-1,-\frac{1}{2}\right],\left[-\frac{1}{4}, 0\right]$, and $\left[\frac{1}{3}, \frac{1}{2}\right]$. The graph of $g$ is shown in figure 4 .

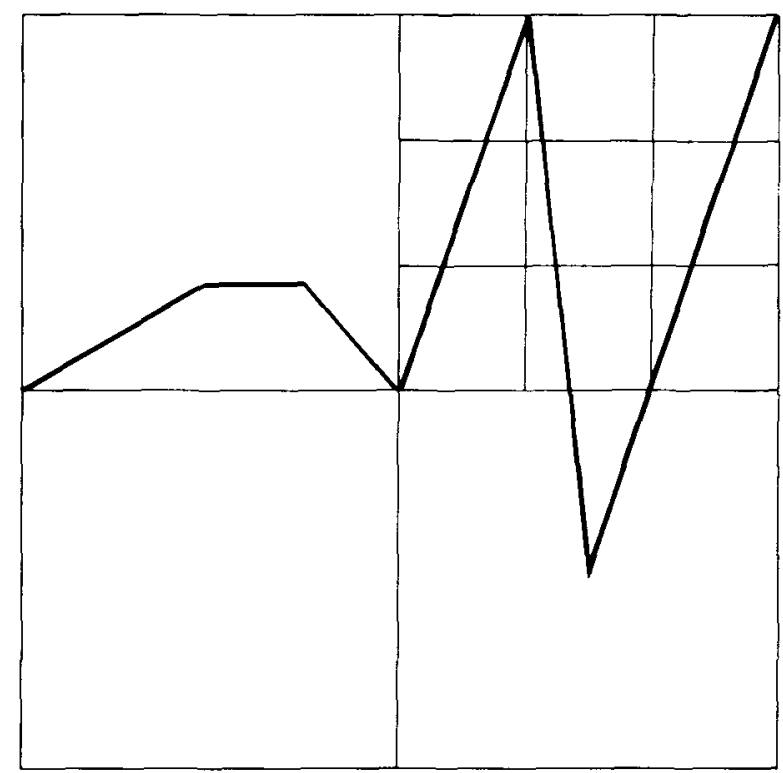

FIGURE 4

It is easy to check that $-\frac{1}{2} \in \Omega(g)-\overline{P(g)}$ and that $\omega\left(-\frac{1}{2}\right)=\omega(y)=C$, and hence that $\omega\left(-\frac{1}{2}\right)$ is not a minimal set.

Notice that $g$ is a particularly simple, in fact piecewise linear, example of a map of the interval with a point in $\Omega-\bar{P}$ which has an infinite orbit. A more complicated example was constructed in [CN, $\S 4]$. 


\section{REFERENCES}

[B] G. D. Birkhoff. Dynamical Systems. Amer. Math. Soc. Colloq. Publ., vol. 9. Amer. Math. Soc.: Providence, RI, 1927.

[C] A. Coppel. Continuous maps on an interval. Xeroxed notes, 1984.

[CN] E. Coven \& Z. Nitecki. Non-wandering sets of powers of maps of the interval. Ergod. Th. \& Dynam. Sys. 1 (1981), 9-31.

[D] J. P. Delahaye. Fonctions admettant des cycles d'order n'importe quelle puissance de 2 et aucun autre cycle. C.R. Acad. Sci. 291 (1980), A323-A325. Addendum A671.

[N1] Z. Nitecki. Periodic and limit orbits and the depth of the center for piecewise monotone interval maps. Proc. Amer. Math. Soc. 80 (1980), 511-514.

[N2] Z. Nitecki. Topological dynamics on the interval, Ergodic theory and dynamical systems II. (College Park, MD, 1979-80), pp. 1-73, Progress in Math. 21. Birkhauser: Boston, 1982.

[S1] A. N. Sarkovskiii. On a theorem of G. D. Birkhoff (Ukrainian, Russian and English summaries). Dopovidi Akad. Nauk Ukrain. RSR Ser. A, (1967), 429-432.

[S2] A. N. Sarkovskǐi. On some properties of discrete dynamical systems, Sur la theorie de l'iteration et ses applications. Colloque international du CNRS, No. 332, Toulouse, 1982.

[SK] A. N. Sarkovskǐi \& H. K. Kenzegulov. On properties of the set of limit points of an iterative sequence of a continuous function (Russian). Voz. Mat. Sb. Vyp. 3 (1965), 343-348.

[X] J.-C. Xiong. $\Omega(f \mid \Omega(f))=\overline{P(f)}$ for every continuous map $f$ of the interval. Kexue Tongbao (English Ed.) 28 (1983), 21-23. 